\title{
Eigenvalues of $s$-type operators on $C(p)$ equipped with a pre-quasi norm
}

\author{
Awad A. Bakery ${ }^{1,2^{*}}$ (D) and Elsayed A.E. Mohamed ${ }^{1,3}$
}

\section{"Correspondence:}

aabhassan@uj.edu.sa;

awad_bakery@yahoo.com;

awad_bakry@hotmail.com

${ }^{1}$ University of Jeddah, College of

Science and Arts at Khulis,

Department of Mathematics,

Jeddah, Saudi Arabia

${ }^{2}$ Department of Mathematics,

Faculty of Science, Ain Shams

University, P.O. Box 1156, Cairo,

11566, Abbassia, Egypt

Full list of author information is

available at the end of the article

\begin{abstract}
We investigate some new topological properties of the multiplication operator on $C(p)$ defined by Lim (Tamkang J. Math. 8(2):213-220, 1977) equipped with the pre-quasi-norm and the pre-quasi-operator ideal formed by this sequence space and s-numbers.
\end{abstract}

MSC: 46B10; 47B10; 46C05; 46E05; 46E15; 46E30

Keywords: Pre-quasi-norm; Multiplication operator; Fredholm operator; Approximable operator; Simple Banch space

\section{Introduction}

Throughout the article, we denote the space of all bounded linear operators from a Banach space $X$ into a Banach space $Y$ by $L(X, Y)$ and if $X=Y$, we write $L(X)$, the space of all complex sequences by $w$, the real numbers $\mathbb{R}$, the complex numbers $\mathbb{C}, \mathbb{N}=\{0,1,2, \ldots\}$, the space of convergent complex sequences to zero by $C_{0}$, the space of bounded complex sequences by $\ell_{\infty}$ and all sequences whose its elements are complex by $\mathbb{C}^{\mathbb{N}}$. For a sequence $\left(p_{n}\right)$ with $\inf _{n} p_{n}>0, \operatorname{Lim}($ see [1]) defined and studied the sequence space $C(p)$ as follows:

$$
\begin{aligned}
& C(p)=\left\{x=\left(x_{n}\right) \in \omega: \rho(\beta x)<\infty \text { for some } \beta>0\right\} \\
& \text { where } \rho(x)=\sum_{n=0}^{\infty}\left(\frac{\sum_{k=2^{n}-1}^{2^{n+1}-2}\left|x_{k}\right|}{2^{n}}\right)^{p_{n}} .
\end{aligned}
$$

The space $C(p)$ is a Banach space with the Luxemburg norm

$$
\|x\|=\inf \left\{\beta>0: \rho\left(\frac{x}{\beta}\right) \leq 1\right\} .
$$

If $\left(p_{n}\right)$ is bounded, we can simply write

$$
C(p)=\left\{\left(x_{i}\right) \in \omega: \sum_{n=0}^{\infty}\left(\frac{\sum_{k=2^{n}-1}^{2^{n+1}}\left|x_{k}\right|}{2^{n}}\right)^{p_{n}}<\infty\right\} .
$$

(c) The Author(s) 2020. This article is licensed under a Creative Commons Attribution 4.0 International License, which permits use sharing, adaptation, distribution and reproduction in any medium or format, as long as you give appropriate credit to the original author(s) and the source, provide a link to the Creative Commons licence, and indicate if changes were made. The images or other third party material in this article are included in the article's Creative Commons licence, unless indicated otherwise in a credit line to the material. If material is not included in the article's Creative Commons licence and your intended use is not permitted by statutory regulation or exceeds the permitted use, you will need to obtain permission directly from the copyright holder. To view a copy of this licence, visit http://creativecommons.org/licenses/by/4.0/. 
Remark 1.1 Taking $p_{n}=p$, for all $n \in \mathbb{N}$, then $C(p)$ is reduced by Lim (see [2]) to ces $_{p}$, he defined and determined its dual spaces and characterize some matrix classes.

The multiplication operators and operator ideals theorems give an importance in functional analysis, since it has numerous applications in fixed point theorem, geometry of Banach spaces, spectral theory and eigenvalue distributions theorem etc. For more details see [3-14]). On sequence spaces, Mursaleen and Noman (see [15]) investigated the compact operators on some difference sequence spaces, Komal and Gupta (see [16]) studied the multiplication operators on Orlicz spaces equipped with the Luxemburg norm and Komal et al. (see [17]) examined the multiplication operators on Cesáro sequence spaces equipped with the Luxemburg norm. Some of operator ideals in the class of Hilbert spaces or Banach spaces are generated by numeric sequence spaces. For example the ideal of compact operators is defined by the space of Kolmogorov numbers and $C_{0}$. Pietsch (see [18]), examined the quasi-ideals generated by $\ell_{p}(0<p<\infty)$ and the approximation numbers. He proved that the ideals of nuclear operators and of Hilbert-Schmidt operators between Hilbert spaces are defined by $\ell_{1}$ and $\ell_{2}$ respectively. He showed that the class of all finite rank operators are dense in the Banach quasi-ideal and the algebra $L\left(\ell_{p}\right)$, where $(1 \leq p<\infty)$ is simple Banach space. Pietsch (see [19]), showed that the quasi-Banach operator ideal formed by the sequence of approximation numbers is small. Makarov and Faried (see [20]) proved that, for any infinite dimensional Banach spaces $X, Y$ and for any $q>p>0, S_{\ell_{p}}^{\mathrm{app}}(X, Y)$ is strictly contained in $S_{\ell_{q}}^{\mathrm{app}}(X, Y)$. Faried and Bakery (see [21]) introduced the concept of pre-quasi-operator ideal which is more general than the usual classes of operator ideal, they studied the operator ideals formed by $s$-numbers, generalized Cesáro and Orlicz sequence spaces $\ell_{M}$, and showed that the operator ideal formed by approximation numbers and the previous sequence spaces is small under certain conditions. The aim of this article to study the concept of pre-quasi-norm on $C(p)$ which is more general than the usual norm, and give the conditions on $C(p)$ equipped with the prequasi-norm to be Banach space. We give the necessity and sufficient conditions on $C(p)$ equipped with the pre-quasi-norm such that the multiplication operator defined on $C(p)$ is a bounded, approximable, invertible, Fredholm and closed range operator. The components of pre-quasi-operator ideal generated by the sequence of $s$-numbers and $C(p)$ is strictly contained for different powers are determined. Furthermore, we give the sufficient conditions on $C(p)$ equipped with a pre-modular such that the pre-quasi-Banach operator ideal constructed by $s$-numbers and $C(p)$ is small, simple and its components is closed. Finally the class of all bounded linear operators, whose sequence of eigenvalues belongs to $C(p)$ is the same as the pre-quasi-operator ideal formed by the sequence of $s$-numbers and $C(p)$.

\section{Definitions and preliminaries}

The spaces of all finite rank, compact and approximable operators on $X$ are denoted by $F(X), L_{c}(X)$ and $\Psi(X)$, respectively.

Lemma 2.1 (see [18]) Let $T \in L(X, Y)$. If $T$ is not approximable, then there are operators $G \in L(X)$ and $B \in L(Y)$ such that $B T G e_{k}=e_{k}$, for all $k \in \mathbb{N}$.

Definition 2.2 (see [18]) A Banach space $X$ is called simple if the algebra $L(X)$ contains one and only one non-trivial closed ideal. 
Theorem 2.3 (see [18]) If $X$ is infinite dimensional Banach space, we have

$$
F(X) \varsubsetneqq \Psi(X) \varsubsetneqq L_{c}(X) \varsubsetneqq L(X) .
$$

Definition 2.4 (see [22]) A bounded linear operator $D: X \rightarrow X$ is called Fredholm if $D$ has closed range, $\operatorname{dim}(\operatorname{ker} D)$ and co-dim $(\operatorname{range} D)$ are finite.

Definition 2.5 (see [23]) A class of linear sequence spaces $X$ is called a special space of sequences (sss) if

(1) $e_{i} \in X$ for all $i \in \mathbb{N}$,

(2) if $u=\left(u_{i}\right) \in w, v=\left(v_{i}\right) \in X$ and $\left|u_{i}\right| \leq\left|v_{i}\right|$ for every $i \in \mathbb{N}$, then $u \in X$ "i.e. $X$ is solid",

(3) if $\left(u_{i}\right)_{i=0}^{\infty} \in X$, then $\left(u_{\left[\frac{i}{2}\right]}\right]_{i=0}^{\infty} \in X$, wherever $\left[\frac{i}{2}\right]$ means the integral part of $\frac{i}{2}$.

Definition 2.6 (see [23]) A subclass of the special space of sequences called a pre-modular (sss) if there is a function $\varrho: X \rightarrow[0, \infty$ [ satisfying the following conditions:

(i) $\varrho(u) \geq 0$ for each $u \in X$ and $\varrho(u)=0 \Leftrightarrow u=\theta$, where $\theta$ is the zero element of $X$,

(ii) there exists $L \geq 1$ such that $\varrho(\beta u) \leq L|\beta| \varrho(u)$ for all $u \in X$, and for any scalar $\beta$,

(iii) for some $K \geq 1, \varrho(u+v) \leq K(\varrho(u)+\varrho(v))$ for every $u, v \in X$,

(iv) if $\left|u_{i}\right| \leq\left|v_{i}\right|$ for all $i \in \mathbb{N}$, then $\varrho\left(\left(u_{i}\right)\right) \leq \varrho\left(\left(v_{i}\right)\right)$,

(v) for some $K_{0} \geq 1, \varrho\left(\left(u_{i}\right)\right) \leq \varrho\left(\left(u_{\left[\frac{i}{2}\right]}\right)\right) \leq K_{0} \varrho\left(\left(u_{i}\right)\right)$,

(vi) the set of all finite sequences is $\varrho$-dense in $X$. This means that, for each $u=\left(u_{i}\right)_{i=o}^{\infty} \in X$, and for each $\varepsilon>0$, there exists $s \in \mathbb{N}$ such that $\varrho\left(\left(u_{i}\right)_{i=s}^{\infty}\right)<\varepsilon$,

(vii) there exists a constant $\xi>0$ such that $\varrho(\beta, 0,0,0, \ldots) \geq \xi|\beta| \varrho(1,0,0,0, \ldots)$ for any $\beta \in \mathbb{R}$.

The concept of pre-quasi-operator ideal which is more general than the usual classes of operator ideal.

Definition 2.7 (see [23]) A function $g: \Omega \rightarrow[0, \infty)$ is said to be a pre-quasi-norm on the ideal $\Omega$ if the following conditions holds:

(1) For all $T \in \Omega(X, Y), g(T) \geq 0$ and $g(T)=0$ if and only if $T=0$,

(2) there exists a constant $M \geq 1$ such that $g(\lambda T) \leq M|\lambda| g(T)$, for all $T \in \Omega(X, Y)$ and $\lambda \in \mathbb{R}$,

(3) there exists a constant $K \geq 1$ such that $g\left(T_{1}+T_{2}\right) \leq K\left[g\left(T_{1}\right)+g\left(T_{2}\right)\right]$, for all $T_{1}, T_{2} \in \Omega(X, Y)$

(4) there exists a constant $C \geq 1$ such that if $T \in L\left(X_{0}, X\right), P \in \Omega(X, Y)$ and $R \in L\left(Y, Y_{0}\right)$ then $g(R P T) \leq C\|R\| g(P)\|T\|$, where $X_{0}$ and $Y_{0}$ are normed spaces.

Definition 2.8 (see [24]) An $s$-number function is a map defined on $L(X, Y)$ which associate to each operator $T \in L(X, Y)$ a non-negative scalar sequence $\left(s_{n}(T)\right)_{n=0}^{\infty}$ assuming that the taking after states are verified:

(a) $\|T\|=s_{0}(T) \geq s_{1}(T) \geq s_{2}(T) \geq \cdots \geq 0$, for $T \in L(X, Y)$,

(b) $s_{m+n-1}\left(T_{1}+T_{2}\right) \leq s_{m}\left(T_{1}\right)+s_{n}\left(T_{2}\right)$ for all $T_{1}, T_{2} \in L(X, Y), m, n \in \mathbb{N}$,

(c) ideal property: $s_{n}(R V T) \leq\|R\| s_{n}(V)\|T\|$ for all $T \in L\left(X_{0}, X\right), V \in L(X, Y)$ and $R \in L\left(Y, Y_{0}\right)$, where $X_{0}$ and $Y_{0}$ are arbitrary Banach spaces,

(d) if $G \in L(X, Y)$ and $\lambda \in \mathbb{R}$, we obtain $s_{n}(\lambda G)=|\lambda| s_{n}(G)$,

(e) rank property: if $\operatorname{rank}(T) \leq n$, then $s_{n}(T)=0$ for each $T \in L(X, Y)$, 
(f) norming property: $s_{r \geq n}\left(I_{n}\right)=0$ or $s_{r<n}\left(I_{n}\right)=1$, where $I_{n}$ represents the unit operator on the $n$-dimensional Hilbert space $\ell_{2}^{n}$.

There are several examples of $s$-numbers, we mention the following:

(1) The $n$th approximation number, denoted by $\alpha_{n}(T)$, is defined by

$$
\alpha_{n}(T)=\inf \{\|T-B\|: B \in L(X, Y) \text { and } \operatorname{rank}(B) \leq n\}
$$

(2) The $n$th Kolmogorov number, denoted by $d_{n}(T)$, is defined by

$$
d_{n}(T)=\inf _{\operatorname{dim} Y \leq n} \sup _{\|x\| \leq 1} \inf _{y \in Y}\|T x-y\|
$$

Notations 2.9 (see [21])

$$
\begin{aligned}
& S_{E}:=\left\{S_{E}(X, Y) ; X \text { and } Y \text { are Banach Spaces }\right\}, \\
& \text { where } S_{E}(X, Y):=\left\{T \in L(X, Y):\left(s_{i}(T)\right)_{i=0}^{\infty} \in E\right\}, \\
& S_{E}^{\text {app }}:=\left\{S_{E}^{\text {app }}(X, Y) ; X \text { and } Y \text { are Banach Spaces }\right\}, \\
& \text { where } S_{E}^{\mathrm{app}}(X, Y):=\left\{T \in L(X, Y):\left(\alpha_{i}(T)\right)_{i=0}^{\infty} \in E\right\}, \\
& S_{E}^{\text {Kol }}:=\left\{S_{E}^{\mathrm{Kol}}(X, Y) ; X \text { and } Y \text { are Banach Spaces }\right\}, \\
& \text { where } S_{E}^{\mathrm{Kol}}(X, Y):=\left\{T \in L(X, Y):\left(d_{i}(T)\right)_{i=0}^{\infty} \in E\right\} .
\end{aligned}
$$

Theorem 2.10 (see [25]) Let $E_{\rho}$ be a pre-modular (sss). Then the linear space $F(X, Y)$ is dense in $S_{E_{\rho}}(X, Y)$, where $g(T)=\rho\left(s_{n}(T)_{n=0}^{\infty}\right)$.

Theorem 2.11 (see [21]) The function $g(T)=\varrho\left(s_{i}(T)\right)_{i=0}^{\infty}$ is a pre-quasi norm on $S_{E_{\varrho}}$, where $E_{\varrho}$ is a pre-modular (sss).

During this paper, we define $e_{n}=\{0,0, \ldots, 1,0,0, \ldots\}$ where 1 appears at the $n$th place for all $n \in \mathbb{N}$, the following well-known inequality (see [26]): $\left|a_{i}+b_{i}\right|^{p_{i}} \leq H\left(\left|a_{i}\right|^{p_{i}}+\left|b_{i}\right|^{p_{i}}\right)$, where $H=\max \left\{1,2^{\sup _{i} p_{i}-1}\right\}, 0 \leq p_{i} \leq \sup _{i} p_{i}<\infty$ and $a_{i}, b_{i} \in \mathbb{C}$ for every $i \in \mathbb{N}$ are used.

\section{Main results}

We give here the concept of pre-quasi-norm on $C(p)$ which is more general than the usual norm, and give the conditions on this sequence space equipped with the pre-quasi-norm to be a Banach space.

Definition 3.1 Let $X$ be special space of sequences (sss). Assume there is a function $\varrho$ : $X \rightarrow[0, \infty[$ satisfying the following conditions:

(i) $\varrho(x) \geq 0$ for each $x \in X$ and $\varrho(x)=0 \Leftrightarrow x=\theta$, where $\theta$ is the zero element of $X$,

(ii) there exists $L \geq 1$ such that $\varrho(\lambda x) \leq L|\lambda| \varrho(x)$ for all $x \in X$, and for any scalar $\lambda$,

(iii) for some $K \geq 1$, we have $\varrho(x+y) \leq K(\varrho(x)+\varrho(y))$ for every $x, y \in X$.

The space $X$ with $\varrho$ is called pre-quasi-normed (sss) and denoted by $X_{\varrho}$, which gives a class more general than the quasi-normed space. If the space $X$ is complete with $\varrho$, then $X_{\varrho}$, is called a pre-quasi-Banach (sss).

We express the following two theorems without verification, since they are clear. 
Theorem 3.2 Every quasi-norm (sss) is pre-quasi-norm (sss).

Theorem 3.3 Every pre-modular (sss) is pre-quasi-normed (sss).

Theorem 3.4 If $\left(p_{n}\right)$ is an increasing bounded sequence with $p_{0}>1$, then the space $(C(p))_{\varrho}$ is a pre-modular Banach (sss), where $\varrho(x)=\sum_{n=0}^{\infty}\left(\frac{\sum_{k=2^{n}-1}^{2^{n+1}}\left|x_{k}\right|}{2^{n}}\right)^{p_{n}}$, for all $x \in C(p)$.

Proof (1-i) let $x, y \in C(p)$. Since $\left(p_{n}\right)$ is bounded, we have

$$
\begin{aligned}
\varrho(x+y) & =\sum_{n=0}^{\infty}\left(\frac{\sum_{k=2^{n}-1}^{2^{n+1}-2}\left|x_{k}+y_{k}\right|}{2^{n}}\right)^{p_{n}} \\
& \leq H\left(\sum_{n=0}^{\infty}\left(\frac{\sum_{k=2^{n}-1}^{2^{n+1}-2}\left|x_{k}\right|}{2^{n}}\right)^{p_{n}}+\sum_{n=0}^{\infty}\left(\frac{\sum_{k=2^{n}-1}^{2^{n+1}-2}\left|y_{k}\right|}{2^{n}}\right)^{p_{n}}\right) \\
& =H(\varrho(x)+\varrho(y))<\infty,
\end{aligned}
$$

then $x+y \in C(p)$.

(1-ii) let $\lambda \in \mathbb{C}, x \in C(p)$ and since $\left(p_{n}\right)$ is bounded, we have

$$
\begin{aligned}
\varrho(\lambda x) & =\sum_{n=0}^{\infty}\left(\frac{\sum_{k=2^{n}-1}^{2^{n+1}-2}\left|\lambda x_{k}\right|}{2^{n}}\right)^{p_{n}} \leq \sup _{n}|\lambda|^{p_{n}} \sum_{n=0}^{\infty}\left(\frac{\sum_{k=2^{n}-1}^{2^{n+1}-2}\left|x_{k}\right|}{2^{n}}\right)^{p_{n}} \\
& =\sup _{n}|\lambda|^{p_{n}} \varrho(x)<\infty .
\end{aligned}
$$

Then $\lambda x \in C(p)$, from (1-i) and (1-ii) $C(p)$ is a linear space.

Since $\left(p_{n}\right)$ is an increasing bounded sequence with $p_{0}>1$, for all $m \in \mathbb{N}$ we have

$$
\varrho\left(e_{m}\right)=\sum_{n=0}^{\infty}\left(\frac{\sum_{k=2^{n}-1}^{2^{n+1}-2}\left(e_{m}\right)_{k}}{2^{n}}\right)^{p_{n}}=\left(\frac{1}{2^{n_{0}}}\right)^{p_{n_{0}}}<\infty,
$$

where $n_{0} \in \mathbb{N}$ is such that $2^{n_{0}}-1 \leq m \leq 2^{n_{0}+1}-2$. Hence $e_{m} \in C(p)$, for all $m \in \mathbb{N}$.

(2) Let $\left|x_{n}\right| \leq\left|y_{n}\right|$ for all $n \in \mathbb{N}$ and $y \in C(p)$. Hence

$$
\varrho(x)=\sum_{n=0}^{\infty}\left(\frac{\sum_{k=2^{n}-1}^{2^{n+1}-2}\left|x_{k}\right|}{2^{n}}\right)^{p_{n}} \leq \sum_{n=0}^{\infty}\left(\frac{\sum_{k=2^{n}-1}^{2^{n+1}-2}\left|y_{k}\right|}{2^{n}}\right)^{p_{n}}=\varrho(y)<\infty
$$

we get $x \in C(p)$.

(3) Let $\left(x_{n}\right) \in C(p)$, we have

$$
\begin{aligned}
\varrho\left(x_{\left[\frac{n}{2}\right]}\right) & =\sum_{n=0}^{\infty}\left(\frac{\sum_{k=2^{n}-1}^{2^{n+1}-2}\left|x_{\left[\frac{k}{2}\right]}\right|}{2^{n}}\right)^{p_{n}} \\
& =\sum_{n=0}^{\infty}\left(\frac{\sum_{k=2^{2 n}-1}^{2^{2 n+1}-2}\left|x_{\left[\frac{k}{2}\right]}\right|}{2^{2 n}}\right)^{p_{2 n}}+\sum_{n=0}^{\infty}\left(\frac{\sum_{k=2^{2 n+1}-1}^{2^{2 n+2}-2}\left|x_{\left[\frac{k}{2}\right]}\right|}{2^{2 n+1}}\right)^{p_{2 n+1}} \\
& \leq \sum_{n=0}^{\infty}\left(\frac{\sum_{k=2^{2 n}-1}^{2^{2 n+1}-2}\left|x_{\left[\frac{k}{2}\right]}\right|}{2^{2 n}}\right)^{p_{n}}+\sum_{n=0}^{\infty}\left(\frac{\sum_{k=2^{2 n+1}-1}^{2^{2 n+2}-2}\left|x_{\left[\frac{k}{2}\right]}\right|}{2^{2 n+1}}\right)^{p_{n}}
\end{aligned}
$$




$$
\begin{aligned}
& \leq \sum_{n=0}^{\infty}\left(\frac{\sum_{k=2^{2 n}-1}^{2^{2 n+1}-2}\left|x_{\left[\frac{k}{2}\right]}\right|}{2^{n}}\right)^{p_{n}}+\sum_{n=0}^{\infty}\left(\frac{\sum_{k=2^{2 n+1}-1}^{2^{2 n+2}-2}\left|x_{\left[\frac{k}{2}\right]}\right|}{2^{n}}\right)^{p_{n}} \\
& \leq\left(2 H^{2}+3 H\right) \sum_{n=0}^{\infty}\left(\frac{\sum_{k=2^{2}-1}^{2^{n+1}-2}\left|x_{k}\right|}{2^{n}}\right)^{p_{n}},
\end{aligned}
$$

then $\left(x_{\left[\frac{n}{2}\right]}\right) \in C(p)$.

(i) Clearly, $\varrho(x) \geq 0$ and $\varrho(x)=0 \Leftrightarrow x=\theta$.

(ii) There is a number $L=\max \left\{1, \sup _{n}|\lambda|^{p_{n}-1}\right\} \geq 1$ with $\varrho(\lambda x) \leq L|\lambda| \varrho(x)$ for all $x \in C(p)$ and $\lambda \in \mathbb{C}$.

(iii) We have the inequality $\varrho(x+y) \leq H(\varrho(x)+\varrho(y))$ for all $x, y \in C(p)$.

(iv) It is clear from (2) that $(C(p))_{Q}$ is solid.

(v) It is clear from (3) that $K_{0} \geq\left(2 H^{2}+3 H\right) \geq 1$.

(vi) It is clear that $\bar{F}=C(p)$.

(vii) There exists a constant $0<\xi \leq|\lambda|^{p_{0}-1}$ such that $\varrho(\lambda, 0,0,0, \ldots) \geq \xi|\lambda| \varrho(1,0,0,0, \ldots)$ for any $\lambda \neq 0$ and $\xi>0$, when $\lambda=0$.

Hence the space $(C(p))_{\varrho}$ is pre-modular (sss). To prove that $(C(p))_{\varrho}$ is a pre-modular Banach (sss), suppose $x^{n}=\left(x_{k}^{n}\right)_{k=0}^{\infty}$ is a Cauchy sequence in $(C(p))_{\varrho}$, then, for every $\varepsilon \in(0,1)$, there exists a number $n_{0} \in \mathbb{N}$ such that, for all $n, m \geq n_{0}$, one has

$$
\varrho\left(x^{n}-x^{m}\right)=\sum_{i=0}^{\infty}\left(\frac{\sum_{j=2^{i}-1}^{2^{i+1}-2}\left|x_{j}^{n}-x_{j}^{m}\right|}{2^{i}}\right)^{p_{i}}<\varepsilon^{\sup _{i} p_{i}} .
$$

Hence, for $n, m \geq n_{0}$ and $i \in \mathbb{N}$, we get

$$
\left|x_{i}^{n}-x_{i}^{m}\right|<\varepsilon
$$

So $\left(x_{i}^{m}\right)$ is a Cauchy sequence in $\mathbb{R}$ for fixed $i \in \mathbb{N}$, this gives $\lim _{m \rightarrow \infty} x_{i}^{m}=x_{i}^{0}$ for fixed $i \in \mathbb{N}$. Hence $\varrho\left(x^{n}-x^{0}\right)<\varepsilon^{\sup _{i} p_{i}}$, for all $n \geq n_{0}$. Finally to prove that $x^{0} \in C(p)$, we have

$$
\varrho\left(x^{0}\right)=\varrho\left(x^{0}-x^{n}+x^{n}\right) \leq H\left(\varrho\left(x^{n}-x^{0}\right)+\varrho\left(x^{n}\right)\right)<\infty
$$

so $x^{0} \in C(p)$. This means that $(C(p))_{\varrho}$ is a pre-modular Banach (sss).

Corollary 3.5 If $1<p<\infty$, then $\left(\operatorname{ces}_{p}\right)_{\varrho}$ is a pre-modular Banach (sss), where $\varrho(x)=$ $\sum_{i=0}^{\infty}\left(\frac{\sum_{j=2^{i}-1}^{2^{i+1}-2}\left|x_{j}\right|}{2^{i}}\right)^{p}$ for all $x \in \operatorname{ces}_{p}$.

\section{Multiplication operator on pre-quasi-normed (sss)}

We define in this section a multiplication operator on $C(p)$ with a pre-quasi-norm and give the necessity and sufficient conditions on the multiplication operator to be bounded, approximable, invertible, Fredholm and closed range operator.

Definition 4.1 Let $\beta \in \mathbb{C}^{\mathbb{N}}$ be a bounded sequence and $E_{\varrho}$ be a pre-quasi-normed (sss), the multiplication operator is defined as $T_{\beta}: E \rightarrow E$, where $T_{\beta} x=\beta x=\left(\beta_{k} x_{k}\right)_{k=0}^{\infty}$, for all $x \in E$. If $T_{\beta}$ is continuous, we call it a multiplication operator induced by $\beta$. 
Theorem 4.2 If $\beta \in \mathbb{C}^{\mathbb{N}}$ and $\left(p_{n}\right)$ is a bounded sequence, then $\beta \in \ell_{\infty}$ if and only if $T_{\beta} \in$ $L\left((C(p))_{\varrho}\right)$, where $\varrho(x)=\sum_{i=0}^{\infty}\left(\frac{\sum_{j=2^{i}-1}^{2^{i+1}-2}\left|x_{j}\right|}{2^{i}}\right) p_{i}$ for all $x \in(C(p))_{\varrho}$.

Proof Let $\beta \in \ell_{\infty}$. Then there exists $C>0$ such that $\left|\beta_{n}\right| \leq C$, for all $n \in \mathbb{N}$. For $x \in(C(p))_{\varrho}$, since $\left(p_{n}\right)$ is a bounded sequence, we have

$$
\begin{aligned}
\varrho\left(T_{\beta} x\right) & =\varrho(\beta x)=\varrho\left(\left(\beta_{k} x_{k}\right)_{k=0}^{\infty}\right)=\varrho\left(\left(\left|\beta_{k}\right|\left|x_{k}\right|\right)_{k=0}^{\infty}\right) \\
& =\sum_{i=0}^{\infty}\left(\frac{\sum_{j=2^{i}-1}^{2^{i+1}-2}\left|\beta_{j}\right|\left|x_{j}\right|}{2^{i}}\right)^{p_{i}} \leq \sum_{i=0}^{\infty}\left(\frac{\sum_{j=2^{i}-1}^{2^{i+1}-2} C\left|x_{j}\right|}{2^{i}}\right)^{p_{i}} \leq A \varrho(x),
\end{aligned}
$$

where $A=\sup _{n} C^{p_{n}}$, this implies that $T_{\beta} \in L\left((C(p))_{\varrho}\right)$.

Conversely, let $T_{\beta} \in L\left((C(p))_{\varrho}\right)$. To show that $\beta \in \ell_{\infty}$. For, if $\beta \notin \ell_{\infty}$, then, for every $n \in \mathbb{N}$, there exists some $i_{n} \in \mathbb{N}$ such that $\beta_{i_{n}}>n$. Now

$$
\varrho\left(T_{\beta} e_{i_{n}}\right)=\varrho\left(\beta e_{i_{n}}\right)=\sum_{i=0}^{\infty}\left(\frac{\sum_{j=2^{i}-1}^{2^{i+1}-2}\left|\beta_{j}\right|\left(e_{i_{n}}\right)_{j}}{2^{i}}\right)^{p_{i}}=\left(\frac{\left|\beta_{i_{n}}\right|}{2^{i_{0}}}\right)^{p_{i_{0}}}>\left(\frac{n}{2^{i_{0}}}\right)^{p_{i_{0}}}=n^{p_{i_{0}}} \varrho\left(e_{i_{n}}\right)
$$

where $i_{0} \in \mathbb{N}$ be such that $2^{i_{0}}-1 \leq i_{n} \leq 2^{i_{0}+1}-2$. This shows that $T_{\beta}$ is not a bounded operator. So, $\beta$ must be a bounded sequence.

Theorem 4.3 Let $\beta \in \mathbb{C}^{\mathbb{N}}$ and $(C(p))_{\varrho}$ be a pre-quasi-normed (sss), where $\varrho(x)=$ $\sum_{i=0}^{\infty}\left(\frac{\sum_{j=2^{i}-1}^{2^{i+1}-2}\left|x_{j}\right|}{2^{i}}\right)^{p_{i}}$, for all $x \in(C(p))_{\varrho}\left|\beta_{n}\right|=1$, for all $n \in \mathbb{N}$ if and only if $T_{\beta}$ is an isometry.

Proof Let $\left|\beta_{n}\right|=1$, for all $n \in \mathbb{N}$. Then

$$
\begin{aligned}
\varrho\left(T_{\beta} x\right) & =\varrho(\beta x)=\varrho\left(\left(\beta_{k} x_{k}\right)_{k=0}^{\infty}\right)=\sum_{i=0}^{\infty}\left(\frac{\sum_{j=2^{i}-1}^{2^{i+1}-2}\left|\beta_{j}\right|\left|x_{j}\right|}{2^{i}}\right)^{p_{i}} \\
& =\sum_{i=0}^{\infty}\left(\frac{\sum_{j=2^{i}-1}^{2^{i+1}-2}\left|x_{j}\right|}{2^{i}}\right)^{p_{i}}=\varrho(x)
\end{aligned}
$$

for all $x \in(C(p))_{\varrho}$. Hence $T_{\beta}$ is an isometry.

Conversely, let $T_{\beta}$ be an isometry and $\left|\beta_{n}\right|<1$ for all $n \in \mathbb{N}$. Then

$$
\begin{aligned}
\varrho\left(T_{\beta} x\right) & =\varrho(\beta x)=\varrho\left(\left(\beta_{k} x_{k}\right)_{k=0}^{\infty}\right)=\sum_{i=0}^{\infty}\left(\frac{\sum_{j=2^{i}-1}^{2^{i+1}-2}\left|\beta_{j}\right|\left|x_{j}\right|}{2^{i}}\right)^{p_{i}} \\
& <\sum_{i=0}^{\infty}\left(\frac{\sum_{j=2^{i}-1}^{2^{i+1}-2}\left|x_{j}\right|}{2^{i}}\right)^{p_{i}}=\varrho(x) .
\end{aligned}
$$

Also if $\left|\beta_{n}\right|>1$, then we get $\varrho(\beta x)>\varrho(x)$. In both cases, we get a contradiction. Hence, $\left|\beta_{n}\right|=1$, for all $n \in \mathbb{N}$.

Theorem 4.4 Let $\beta \in \mathbb{C}^{\mathbb{N}},\left(p_{n}\right)$ be a bounded sequence and $T_{\beta} \in L\left((C(p))_{\varrho}\right)$, where $\varrho(x)=$ $\sum_{i=0}^{\infty}\left(\frac{\sum_{j=2^{i}-1}^{2^{i+1}-2}\left|x_{j}\right|}{2^{i}}\right)^{p_{i}}$ for all $x \in(C(p))_{\varrho}$. Then $T_{\beta} \in \Psi\left((C(p))_{\varrho}\right)$ if and only if $\left(\beta_{n}\right)_{n=0}^{\infty} \in C_{0}$. 
Proof Let $T_{\beta}$ be an approximable operator. Hence $T_{\beta}$ is compact operator. We have to show that $\left(\beta_{n}\right)_{n=0}^{\infty} \in C_{0}$. For if this is not true, then there exists $\delta>0$ such that $B_{\delta}=\{r \in \mathbb{N}$ : $\left.\left|\beta_{r}\right| \geq \delta\right\}$ is an infinite set. Let $d_{1}, d_{2}, \ldots, d_{n}, \ldots$ be in $B_{\delta}$. Then $\left\{e_{d_{n}}: d_{n} \in B_{\delta}\right\}$ is an infinite bounded set in $(C(p))_{\varrho}$. We have

$$
\begin{aligned}
\varrho\left(T_{\beta} e_{d_{n}}-T_{\beta} e_{d_{m}}\right) & =\varrho\left(\beta e_{d_{n}}-\beta e_{d_{m}}\right)=\varrho\left(\left(\beta_{k}\left(\left(e_{d_{n}}\right)_{k}-\left(e_{p_{m}}\right)_{k}\right)\right)_{k=0}^{\infty}\right) \\
& =\sum_{i=0}^{\infty}\left(\frac{\sum_{j=2^{i}-1}^{2^{i+1}-2}\left|\beta_{j}\left(\left(e_{d_{n}}\right)_{j}-\left(e_{p_{m}}\right)_{j}\right)\right|}{2^{i}}\right)^{p_{i}} \\
& \geq \sum_{i=0}^{\infty}\left(\frac{\sum_{j=2^{i}-1}^{2^{i+1}-2}\left|\delta\left(\left(e_{d_{n}}\right)_{j}-\left(e_{p_{m}}\right)_{j}\right)\right|}{2^{i}}\right)^{p_{i}} \\
& \geq\left(\inf _{n} \delta^{p_{n}}\right) \sum_{i=0}^{\infty}\left(\frac{\sum_{j=2^{i}-1}^{2^{i+1}-2}\left|\left(\left(e_{d_{n}}\right)_{j}-\left(e_{p_{m}}\right)_{j}\right)\right|}{2^{i}}\right)^{p_{i}} \\
& =\left(\inf _{n} \delta^{p_{n}}\right) \varrho\left(e_{d_{n}}-e_{d_{m}}\right),
\end{aligned}
$$

for all $d_{n}, d_{m} \in B_{\delta}$. This proves $\left\{e_{d_{n}}: d_{n} \in B_{\delta}\right\}$ is a bounded sequence, which cannot have a convergent subsequence under $T_{\beta}$. This shows that $T_{\beta}$ cannot be a compact, hence is not approximable operator, which is a contradiction. Hence, $\lim _{n \rightarrow \infty} \beta_{n}=0$.

Conversely, suppose $\lim _{n \rightarrow \infty} \beta_{n}=0$. Then, for every $\delta>0$, the set $B_{\delta}=\left\{n \in \mathbb{N}:\left|\beta_{n}\right| \geq \delta\right\}$ is finite. Then

$$
\left((C(p))_{\varrho}\right)_{B_{\delta}}=\left\{x=\left(x_{n}\right) \in(C(p))_{\varrho}: n \in B_{\delta}\right\}
$$

is a finite dimensional space for each $\delta>0$. Therefore, $T_{\beta} \mid\left((C(p))_{\varrho}\right)_{B_{\delta}}$ is a finite rank operator. For each $n \in \mathbb{N}$, define $\beta_{n}: \mathbb{N} \rightarrow \mathbb{C}$ by

$$
\left(\beta_{n}\right)_{m}= \begin{cases}\beta_{m}, & m \in B_{\frac{1}{n}} \\ 0, & \text { otherwise }\end{cases}
$$

Clearly, $T_{\beta_{n}}$ is a finite rank operator as the space $\left((C(p))_{\varrho}\right)_{B_{\frac{1}{n}}}$ is finite dimensional for each $n \in \mathbb{N}$. Now, by using $\left(p_{n}\right)$ is a bounded sequence, we have

$$
\begin{aligned}
\varrho\left(\left(T_{\beta}-T_{\beta_{n}}\right) x\right)= & \varrho\left(\left(\left(\beta_{m}-\left(\beta_{n}\right)_{m}\right) x_{m}\right)_{m=0}^{\infty}\right) \\
= & \sum_{i=0, i \in B_{\frac{1}{n}}}^{\infty}\left(\frac{\sum_{j=2^{i}-1}^{2^{i+1}-2}\left|\left(\beta_{j}-\left(\beta_{n}\right)_{j}\right) x_{j}\right|}{2^{i}}\right)^{p_{i}} \\
& +\sum_{i=0, i \notin B_{\frac{1}{n}}}^{\infty}\left(\frac{\sum_{j=2^{i}-1}^{2^{i+1}-2}\left|\left(\beta_{j}-\left(\beta_{n}\right)_{j}\right) x_{j}\right|}{2^{i}}\right)^{p_{i}}
\end{aligned}
$$




$$
\begin{aligned}
& =\sum_{i=0, i \in B_{\frac{1}{n}}}^{\infty}\left(\frac{\sum_{j=2^{i}-1, j \notin B_{\frac{1}{n}}}^{2^{i+1}-2}\left|\beta_{j} x_{j}\right|}{2^{i}}\right)^{p_{i}}+\sum_{i=0, i \notin B}^{\infty}\left(\frac{\sum_{\frac{1}{n}}^{2_{j=2^{i}-1, j \notin B_{\frac{1}{n}}}^{i+2}\left|\beta_{j} x_{j}\right|}}{2^{i}}\right)^{p_{i}} \\
& <\left(\frac{1}{n}\right)^{\inf _{n} p_{n}} \sum_{i=0}^{\infty}\left(\frac{\sum_{j=2^{i}-1}^{2^{i+1}-2}\left|x_{j}\right|}{2^{i}}\right)^{p_{i}}=\left(\frac{1}{n}\right)^{\inf _{n} p_{n}} \varrho(x) .
\end{aligned}
$$

This proves that $\left\|T_{\beta}-T_{\beta_{n}}\right\| \leq\left(\frac{1}{n}\right)^{\inf _{n} p_{n}}$ and that $T_{\beta}$ is a limit of finite rank operators and hence, $T_{\beta}$ is an approximable operator.

Theorem 4.5 Let $\beta \in \mathbb{C}^{\mathbb{N}},\left(p_{n}\right)$ be a bounded sequence and $T_{\beta} \in L\left((C(p))_{\varrho}\right)$, where $\varrho(x)=$ $\sum_{i=0}^{\infty}\left(\frac{\sum_{j=2^{i}-1}^{2^{i+1}-2}\left|x_{j}\right|}{2^{i}}\right)^{p_{i}}$ for all $x \in(C(p))_{\varrho}$. Then $T_{\beta} \in L_{c}\left((C(p))_{\varrho}\right)$ if and only if $\left(\beta_{n}\right)_{n=0}^{\infty} \in C_{0}$.

Proof It is easy so omitted.

Corollary 4.6 Let $\left(p_{n}\right)$ be a bounded sequence, we have

$$
L_{c}\left((C(p))_{\varrho}\right) \varsubsetneqq L\left((C(p))_{\varrho}\right) \text {, }
$$

where $\varrho(x)=\sum_{i=0}^{\infty}\left(\frac{\sum_{j=2^{i}-2}^{2^{i+1}-2}\left|x_{j}\right|}{2^{i}}\right)^{p_{i}}$ for all $x \in C(p)$.

Proof Since the identity operator $I$ on $(C(p))_{\varrho}$ is a multiplication operator induced by the sequence $\beta=(1,1, \ldots)$, we have $I \notin L_{c}\left((C(p))_{\varrho}\right)$ and $I \in L\left((C(p))_{\varrho}\right)$.

Theorem 4.7 Let $(C(p))_{\varrho}$ be a pre-quasi-Banach (sss), where $\varrho(x)=\sum_{i=0}^{\infty}\left(\frac{\sum_{j=2^{i}-1}^{2^{i+1}-2}\left|x_{j}\right|}{2^{i}}\right)^{p_{i}}$ for all $x \in C(p)$ and $T_{\beta} \in L\left((C(p))_{\varrho}\right)$. Then $\beta$ is bounded away from zero on $\mathbb{N} \backslash \operatorname{ker}(\beta):=\operatorname{ker}(\beta)^{c}$ if and only if $T_{\beta}$ has closed range.

Proof Let $\beta$ be bounded away from zero on $\operatorname{ker}(\beta)^{c}$. Then there exists $\epsilon>0$ such that $\left|\beta_{n}\right| \geq \epsilon$, for all $n \in \operatorname{ker}(\beta)^{c}$. We have to prove that range $\left(T_{\beta}\right)$ is closed. Let $f$ be a limit point of range $\left(T_{\beta}\right)$. Then there exists a sequence $T_{\beta} x_{n}$ in $(C(p))_{\varrho}$, for all $n \in \mathbb{N}$ such that $\lim _{n \rightarrow \infty} T_{\beta} x_{n}=f$. Clearly, the sequence $T_{\beta} x_{n}$ is a Cauchy sequence. Now, since $\varrho$ is nondecreasing, we have

$$
\begin{aligned}
& \varrho\left(T_{\beta} x_{n}-T_{\beta} x_{m}\right) \\
& =\varrho\left(\left(\beta_{j}\left(x_{n}\right)_{j}-\beta_{j}\left(x_{m}\right)_{j}\right)_{j=0}^{\infty}\right)=\sum_{i=0}^{\infty}\left(\frac{\sum_{j=2^{i}-1}^{2^{i+1}-2}\left|\beta_{j}\left(x_{n}\right)_{j}-\beta_{j}\left(x_{m}\right)_{j}\right|}{2^{i}}\right)^{p_{i}} \\
& =\sum_{i=0, i \in \operatorname{ker}(\beta)^{c}}^{\infty}\left(\frac{\sum_{j=2^{i}-1}^{2^{i+1}-2}\left|\beta_{j}\left(x_{n}\right)_{j}-\beta_{j}\left(x_{m}\right)_{j}\right|}{2^{i}}\right)^{p_{i}} \\
& \quad+\sum_{i=0, i \notin \operatorname{ker}(\beta)^{c}}^{\infty}\left(\frac{\sum_{j=2^{i}-1}^{2^{i+1}-2}\left|\beta_{j}\left(x_{n}\right)_{j}-\beta_{j}\left(x_{m}\right)_{j}\right|}{2^{i}}\right)^{p_{i}}
\end{aligned}
$$




$$
\begin{aligned}
& \geq \sum_{i=0, i \in \operatorname{ker}(\beta)^{c}}^{\infty}\left(\frac{\sum_{j=2^{i}-1}^{2^{i+1}-2}\left|\beta_{j}\left(x_{n}\right)_{j}-\beta_{j}\left(x_{m}\right)_{j}\right|}{2^{i}}\right)^{p_{i}}=\sum_{i=0}^{\infty}\left(\frac{\sum_{j=2^{i}-1}^{2^{i+1}-2}\left|\beta_{j}\left(y_{n}\right)_{j}-\beta_{j}\left(y_{m}\right)_{j}\right|}{2^{i}}\right)^{p_{i}} \\
& >\sum_{i=0}^{\infty}\left(\frac{\sum_{j=2^{i}-1}^{2^{i+1}-2}\left|\epsilon\left(y_{n}\right)_{j}-\epsilon\left(y_{m}\right)_{j}\right|}{2^{i}}\right)^{p_{i}}=\varrho\left(\epsilon\left(y_{n}-y_{m}\right)\right),
\end{aligned}
$$

where

$$
\left(y_{n}\right)_{k}= \begin{cases}\left(x_{n}\right)_{k}, & k \in \operatorname{ker}(\beta)^{c}, \\ 0, & k \notin \operatorname{ker}(\beta)^{c} .\end{cases}
$$

This proves that $\left(y_{n}\right)$ is a Cauchy sequence in $(C(p))_{\varrho}$. But $(C(p))_{\varrho}$ is complete. Therefore, there exists $x \in(C(p))_{\varrho}$ such that $\lim _{n \rightarrow \infty} y_{n}=x$. In view of the continuity of $T_{\beta}$, hence $\lim _{n \rightarrow \infty} T_{\beta} y_{n}=T_{\beta} x$. But $\lim _{n \rightarrow \infty} T_{\beta} x_{n}=\lim _{n \rightarrow \infty} T_{\beta} y_{n}=f$. Therefore, $T_{\beta} x=f$. Hence $f \in$ range $\left(T_{\beta}\right)$. This proves that $T_{\beta}$ has closed range.

Conversely, suppose that $T_{\beta}$ has closed range. Then $T_{\beta}$ is bounded away from zero on $\left((C(p))_{\varrho}\right)_{\operatorname{ker}(\beta) c}$. That is, there exists $\epsilon>0$ such that $\varrho\left(T_{\beta} x\right) \geq \varrho(\epsilon x)$, for all $x \in$ $\left((C(p))_{\varrho}\right)_{\operatorname{ker}(\beta)^{c}}$. Let $D=\left\{k \in \operatorname{ker}(\beta)^{c}:\left|\beta_{k}\right|<\epsilon\right\}$. If $D \neq \phi$, then, for $n_{0} \in D$, since $\varrho$ is nondecreasing, we have

$$
\begin{aligned}
\varrho\left(T_{\beta} e_{n_{0}}\right) & \left.=\varrho\left(\left(\beta_{k}\left(e_{n_{0}}\right)_{k}\right)\right)_{k=0}^{\infty}\right)=\varrho\left(\left(\left|\beta_{k}\right|\left|\left(e_{n_{0}}\right)_{k}\right|\right)_{k=0}^{\infty}\right)=\sum_{i=0}^{\infty}\left(\frac{\sum_{j=2^{i}-1}^{2^{i+1}}\left|\beta_{j}\right|\left|\left(e_{n_{0}}\right)_{j}\right|}{2^{i}}\right)^{p_{i}} \\
& <\sum_{i=0}^{\infty}\left(\frac{\sum_{j=2^{i}-1}^{2^{i+1}-2} \epsilon\left|\left(e_{n_{0}}\right)_{j}\right|}{2^{i}}\right)^{p_{i}}=\varrho\left(\epsilon e_{n_{0}}\right),
\end{aligned}
$$

which is a contradiction. Hence, $D=\phi$ so that $\left|\beta_{k}\right| \geq \epsilon$, for all $k \in \operatorname{ker}(\beta)^{c}$. This proves the theorem.

Theorem 4.8 Let $\beta \in \mathbb{C}^{\mathbb{N}}$ and $(C(p))_{\varrho}$ be a pre-quasi-Banach (sss), where $\varrho(x)=$ $\sum_{i=0}^{\infty}\left(\frac{\sum_{j=2^{i}-1}^{2^{i+1}-2}\left|x_{j}\right|}{2^{i}}\right)^{p_{i}}$ for all $x \in C(p)$. There exist $a>0$ and $A>0$ such that $a<\beta_{n}<A$; for all $n \in \mathbb{N}$ if and only if $T_{\beta} \in L\left((C(p))_{\varrho}\right)$ is invertible.

Proof Let the condition be true. Define $\eta \in \mathbb{C}^{\mathbb{N}}$ by $\eta_{n}=\frac{1}{\beta_{n}}$. Then by Theorem 4.2, $T_{\beta}$ and $T_{\eta}$ are bounded linear operators. Also $T_{\beta} \cdot T_{\eta}=T_{\eta} \cdot T_{\beta}=I$. Hence, $T_{\beta}$ is the inverse of $T_{\eta}$. Conversely, let $T_{\beta}$ be invertible. Then range $\left(T_{\beta}\right)=\left((C(p))_{\varrho}\right)_{\mathbb{N}}$. Therefore, $\operatorname{range}\left(T_{\beta}\right)$ is closed. Hence, by Theorem 4.7, there exists $a>0$ such that $\left|\beta_{n}\right| \geq a$, for all $n \in \operatorname{ker}(\beta)^{c}$. Now $\operatorname{ker}(\beta)=\phi$; otherwise $\beta_{n_{0}}=0$, for some $n_{0} \in \mathbb{N}$, in which case $e_{n_{0}} \in \operatorname{ker}\left(T_{\beta}\right)$ which is a contradiction, since $\operatorname{ker}\left(T_{\beta}\right)$ is trivial. Hence, $\left|\beta_{n}\right| \geq a$, for all $n \in \mathbb{N}$. Since $T_{\beta}$ is bounded, so by Theorem 4.2, there exists $A>0$ such that $\left|\beta_{n}\right| \leq A$, for all $n \in \mathbb{N}$. Thus, we have proved that $a \leq\left|\beta_{n}\right| \leq A$, for all $n \in \mathbb{N}$.

Theorem 4.9 Let $(C(p))_{\varrho}$ be a pre-quasi-Banach $($ sss $)$, where $\varrho(x)=\sum_{i=0}^{\infty}\left(\frac{\sum_{j=2^{i}-1}^{2^{i+1}-2}\left|x_{j}\right|}{2^{i}}\right)^{p_{i}}$ for all $x \in C(p)$ and $T_{\beta} \in L\left((C(p))_{\varrho}\right)$. Then $T_{\beta}$ is a Fredholm operator if and only if:

(i) $\operatorname{ker}(\beta)$ is a finite subset of $\mathbb{N}$.

(ii) $\left|\beta_{n}\right| \geq \epsilon$, for all $n \in \operatorname{ker}(\beta)^{c}$. 
Proof Let $T_{\beta}$ be Fredholm. If $\operatorname{ker}(\beta)$ is an infinite subset of $\mathbb{N}$, then $e_{n} \in \operatorname{ker}\left(T_{\beta}\right)$, for all $n \in \operatorname{ker}(\beta)$. But $e_{n}$ 's are linearly independent, which shows that $\operatorname{ker}\left(T_{\beta}\right)$ is an infinite dimensional which is a contradiction. Hence, $\operatorname{ker}(\beta)$ must be a finite subset of $\mathbb{N}$. The condition (ii) comes from Theorem 4.7. Conversely, If the conditions (i) and (ii) are true, then we prove that $T_{\beta}$ is Fredholm. By Theorem 4.7, the condition (ii) implies that $T_{\beta}$ has closed range. The condition (i) implies that $\operatorname{ker}\left(T_{\beta}\right)$ and $\left(\operatorname{range}\left(T_{\beta}\right)\right)^{c}$ are finite dimensional. This proves that $T_{\beta}$ is Fredholm.

\section{Completeness and closedness of the pre-quasi-ideal components}

We give here the sufficient conditions on $C(p)$ such that the components of pre-quasioperator ideal $S_{C(p)}$ are complete.

Theorem 5.1 If $X, Y$ are Banach spaces and $\left(p_{n}\right)$ is an increasing bounded sequence with $p_{0}>1$, then $\left(S_{(C(p))_{\varrho}}, g\right)$, where $\varrho(x)=\sum_{i=0}^{\infty}\left(\frac{\sum_{j=2^{i}-1}^{2^{i+1}-2}\left|x_{j}\right|}{2^{i}}\right)^{p_{i}}$ for all $x \in C(p)$ and $g(T)=$ $\varrho\left(\left(s_{n}(T)\right)_{n=0}^{\infty}\right)$ is a pre-quasi-Banach operator ideal.

Proof Since $(C(p))_{\varrho}$ is a pre-modular (sss) by Theorem 3.4, then from Theorem 2.11, the function $g(T)=\varrho\left(\left(s_{n}(T)\right)_{n=0}^{\infty}\right)$ is a pre-quasi-norm on $S_{(C(p))_{e}}$. Let $\left(T_{i}\right)$ be a Cauchy sequence in $S_{(C(p))_{e}}(X, Y)$, since $L(X, Y) \supseteq S_{(C(p))_{e}}(X, Y)$, we get

$$
g\left(T_{i}-T_{j}\right)=\sum_{n=0}^{\infty}\left(\frac{\sum_{m=2^{n}-1}^{2^{n+1}-2} s_{m}\left(T_{i}-T_{j}\right)}{2^{n}}\right)^{p_{n}} \geq\left(\left\|T_{i}-T_{j}\right\|\right)^{p_{0}},
$$

then $\left(T_{j}\right)_{j \in \mathbb{N}}$ is a Cauchy sequence in $L(X, Y)$. While the space $L(X, Y)$ is a Banach space, there exists $T \in L(X, Y)$ with $\lim _{i \rightarrow \infty}\left\|T_{i}-T\right\|=0$ and while $\left(s_{n}\left(T_{i}\right)\right)_{n=0}^{\infty} \in(C(p))_{\varrho}$ for each $i \in \mathbb{N}$, using definition (2.6) conditions (iii), (iv) and $\varrho$ being continuous at $\theta$, we get

$$
\begin{aligned}
g(T) & =\sum_{i=0}^{\infty}\left(\frac{\sum_{j=2^{i}-1}^{2^{i+1}} s_{j}(T)}{2^{i}}\right)^{p_{i}}=\sum_{i=0}^{\infty}\left(\frac{\sum_{j=2^{i}-1}^{2^{i+1}-2} s_{j}\left(T-T_{m}+T_{m}\right)}{2^{i}}\right)^{p_{i}} \\
& \leq K \sum_{i=0}^{\infty}\left(\frac{\sum_{j=2^{i}-1}^{2^{i+1}-2} s_{\left[\frac{j}{2}\right]}\left(T-T_{m}\right)}{2^{i}}\right)^{p_{i}}+K \sum_{i=0}^{\infty}\left(\frac{\sum_{j=2^{i}-1}^{2^{i+1}-2} s_{\left[\frac{j}{2}\right]}\left(T_{m}\right)}{2^{i}}\right)^{p_{i}} \\
& \leq K \sum_{i=0}^{\infty}\left(\frac{\sum_{j=2^{i}-1}^{2^{i+1}-2}\left\|T_{m}-T\right\|}{2^{i}}\right)^{p_{i}}+K K_{0} \sum_{i=0}^{\infty}\left(\frac{\sum_{j=2^{i}-1}^{2^{i+1}-2} s_{j}\left(T_{m}\right)}{2^{i}}\right)^{p_{i}}<\varepsilon,
\end{aligned}
$$

we have $\left(s_{n}(T)\right)_{n=0}^{\infty} \in(C(p))_{\varrho}$, then $T \in S_{(C(p))_{\varrho}}(X, Y)$.

We give here the sufficient conditions on $C(p)$ such that the components of pre-quasioperator ideal $S_{C(p)}$ are closed.

Theorem 5.2 If $X, Y$ are normed spaces, $\left(p_{n}\right)$ is an increasing bounded sequence with $p_{0}>1$, then $\left(S_{(C(p))_{\varrho}}, g\right)$ is a pre-quasi-closed operator ideal, where $\varrho(x)=\sum_{i=0}^{\infty}\left(\frac{\sum_{j=2^{i}-1}^{2^{i+1}-2}\left|x_{j}\right|}{2^{i}}\right)^{p_{i}}$ for all $x \in C(p)$ and $g(T)=\varrho\left(\left(s_{n}(T)\right)_{n=0}^{\infty}\right)$.

Proof Since $C(p)$ is a pre-modular (sss) by Theorem 3.4, from Theorem 2.11, the function $g(T)=\varrho\left(\left(s_{n}(T)\right)_{n=0}^{\infty}\right)$ is a pre-quasi-norm on $S_{(C(p))_{e}}$. Let $T_{m} \in S_{(C(p))_{\varrho}}(X, Y)$ for all $m \in \mathbb{N}$ 
and $\lim _{m \rightarrow \infty} g\left(T_{m}-T\right)=0$, since $L(X, Y) \supseteq S_{(C(p))_{\ell}}(X, Y)$, we get

$$
g\left(T-T_{j}\right)=\sum_{n=0}^{\infty}\left(\frac{\sum_{m=2^{n}-1}^{2^{n+1}-2} s_{m}\left(T-T_{j}\right)}{2^{n}}\right)^{p_{n}} \geq\left(\left\|T-T_{j}\right\|\right)^{p_{0}},
$$

then $\left(T_{j}\right)_{j \in \mathbb{N}}$ is a convergent sequence in $L(X, Y)$. While $\left(s_{n}\left(T_{j}\right)\right)_{n=0}^{\infty} \in(C(p))_{\varrho}$ for each $j \in \mathbb{N}$, hence using definition (2.6) conditions (iii), (iv) and $\varrho$ is continuous at $\theta$, we obtain

$$
\begin{aligned}
g(T) & =\sum_{i=0}^{\infty}\left(\frac{\sum_{j=2^{i}-1}^{2^{i+1}} s_{j}(T)}{2^{i}}\right)^{p_{i}}=\sum_{i=0}^{\infty}\left(\frac{\sum_{j=2^{i}-1}^{2^{i+1}-2} s_{j}\left(T-T_{m}+T_{m}\right)}{2^{i}}\right)^{p_{i}} \\
& \leq K \sum_{i=0}^{\infty}\left(\frac{\sum_{j=2^{i}-1}^{2^{i+1}-2} s_{\left[\frac{j}{2}\right]}\left(T-T_{m}\right)}{2^{i}}\right)^{p_{i}}+K \sum_{i=0}^{\infty}\left(\frac{\sum_{j=2^{i}-1}^{2^{i+1}-2} s_{\left[\frac{j}{2}\right]}\left(T_{m}\right)}{2^{i}}\right)^{p_{i}} \\
& \leq K \sum_{i=0}^{\infty}\left(\frac{\sum_{j=2^{i}-1}^{2^{i+1}-2}\left\|T_{m}-T\right\|}{2^{i}}\right)^{p_{i}}+K K_{0} \sum_{i=0}^{\infty}\left(\frac{\sum_{j=2^{i}-1}^{2^{i+1}-2} s_{j}\left(T_{m}\right)}{2^{i}}\right)^{p_{i}}<\varepsilon,
\end{aligned}
$$

we have $\left(s_{n}(T)\right)_{n=0}^{\infty} \in(C(p))_{\varrho}$, then $T \in S_{(C(p))_{\varrho}}(X, Y)$.

\section{Smallness and simpleness of the pre-quasi-Banach operator ideal}

We give here the sufficient conditions on $C(p)$ such that the pre-quasi-operator ideal formed by the sequence of $s$-numbers and this sequence space is strictly contained for different powers.

Theorem 6.1 Let $X, Y$ be infinite dimensional Banach spaces and for any $1<p_{n}<q_{n}$ for all $n \in \mathbb{N}$, it is true that

$$
S_{C(p)}(X, Y) \varsubsetneqq S_{C(q)}(X, Y) \varsubsetneqq L(X, Y) .
$$

Proof Let the conditions be satisfied, if $T \in S_{C(p)}(X, Y)$, then $\left(s_{j}(T)\right) \in C(p)$. We have

$$
\sum_{i=0}^{\infty}\left(\frac{\sum_{j=2^{i}-1}^{2^{i+1}-2} s_{j}(T)}{2^{i}}\right)^{q_{i}}<\sum_{i=0}^{\infty}\left(\frac{\sum_{j=2^{i}-1}^{2^{i+1}-2} s_{j}(T)}{2^{i}}\right)^{p_{i}}<\infty,
$$

hence $T \in S_{C(q)}(X, Y)$. Next, if we take $\left(s_{j}(T)\right)_{j=0}^{\infty}$ such that

$$
\sum_{j=2^{i}-1}^{2^{i+1}-2} s_{j}(T)=2^{i}(i+1)^{-\frac{1}{p_{i}}}
$$

one can find $T \in L(X, Y)$ with

$$
\sum_{i=0}^{\infty}\left(\frac{\sum_{j=2^{i}-1}^{2^{i+1}-2} s_{j}(T)}{2^{i}}\right)^{p_{i}}=\sum_{i=0}^{\infty} \frac{1}{i+1}=\infty
$$


and

$$
\sum_{i=0}^{\infty}\left(\frac{\sum_{j=2^{i}-1}^{2^{i+1}-2} s_{j}(T)}{2^{i}}\right)^{q_{i}}=\sum_{i=0}^{\infty}\left(\frac{1}{i+1}\right)^{\frac{q_{i}}{p_{i}}}<\infty
$$

Hence $T$ does not belong to $S_{C(p)}(X, Y)$ and $T \in S_{C(q)}(X, Y)$.

It is easy to verify that $S_{C(q)}(X, Y) \subset L(X, Y)$. Next, if we take $\left(s_{j}(T)\right)_{j=0}^{\infty}$ such that $\sum_{j=2^{i}-1}^{2^{i+1}-2} s_{j}(T)=2^{i}(i+1)^{-\frac{1}{q_{i}}}$. One can find $T \in L(X, Y)$ such that $T$ does not belong to $S_{C(q)}(X, Y)$. This completes the proof.

Corollary 6.2 For any infinite dimensional Banach spaces $X, Y$ and $1<p<q<\infty$, then

$$
S_{\operatorname{ces}_{p}}(X, Y) \varsubsetneqq S_{\operatorname{ces}_{q}}(X, Y) \varsubsetneqq L(X, Y) .
$$

In this part, we give the conditions for which the pre-quasi-Banach operator ideal $S_{C(p)}^{\text {app }}$ is small.

Theorem 6.3 If $\left(p_{n}\right)$ is an increasing bounded sequence with $p_{0}>1$, then the pre-quasiBanach operator ideal $S_{C(p)}^{\mathrm{app}}$ is small.

Proof Let $\left(p_{n}\right)$ be an increasing bounded sequence with $p_{0}>1$, take $\epsilon=\left(\sum_{i=0}^{\infty} 2^{-i p_{i}}\right)^{\frac{1}{\sup _{i} p_{i}}}$. By using Theorems 3.3 and 3.4. Then $\left(S_{C(p)}^{\text {app }}, g\right)$, where

$$
g(T)=\frac{1}{\epsilon}\left[\sum_{i=0}^{\infty}\left(\frac{\sum_{j=2^{i}-1}^{2^{i+1}-2} s_{j}(T)}{2^{i}}\right)^{p_{i}}\right]^{\frac{1}{\sup _{i} p_{i}}}
$$

is a pre-quasi-Banach operator ideal. Let $X$ and $Y$ be any two Banach spaces. Suppose that $S_{C(p)}^{\text {app }}(X, Y)=L(X, Y)$, then there exists a constant $C>0$ such that $g(T) \leq C\|T\|$ for all $T \in L(X, Y)$. Suppose that $X$ and $Y$ be infinite dimensional Banach spaces. Hence by Dvoretzky's theorem (see [27]) for $n \in \mathbb{N}$, we have quotient spaces $X / N_{n}$ and subspaces $M_{n}$ of $Y$ which can be mapped onto $\ell_{2}^{n}$ by isomorphisms $H_{n}$ and $A_{n}$ such that $\left\|H_{n}\right\|\left\|H_{n}^{-1}\right\| \leq 2$ and $\left\|A_{n}\right\|\left\|A_{n}^{-1}\right\| \leq 2$. Let $I_{n}$ be the identity map on $\ell_{2}^{n}, Q_{n}$ be the quotient map from $X$ onto $X / N_{n}$ and $J_{n}$ be the natural embedding map from $M_{n}$ into $Y$. Let $\gamma_{j}$ be the Bernstein numbers (see [28]) then

$$
\begin{aligned}
1 & =\gamma_{j}\left(I_{n}\right)=\gamma_{j}\left(A_{n} A_{n}^{-1} I_{n} H_{n} H_{n}^{-1}\right) \leq\left\|A_{n}\right\| \gamma_{j}\left(A_{n}^{-1} I_{n} H_{n}\right)\left\|H_{n}^{-1}\right\| \\
& =\left\|A_{n}\right\| \gamma_{j}\left(J_{n} A_{n}^{-1} I_{n} H_{n}\right)\left\|H_{n}^{-1}\right\| \leq\left\|A_{n}\right\| d_{j}\left(J_{n} A_{n}^{-1} I_{n} H_{n}\right)\left\|H_{n}^{-1}\right\| \\
& =\left\|A_{n}\right\| d_{j}\left(J_{n} A_{n}^{-1} I_{n} H_{n} Q_{n}\right)\left\|H_{n}^{-1}\right\| \leq\left\|A_{n}\right\| \alpha_{j}\left(J_{n} A_{n}^{-1} I_{n} H_{n} Q_{n}\right)\left\|H_{n}^{-1}\right\|,
\end{aligned}
$$

for $0 \leq i \leq n$. Now

$$
\begin{gathered}
\sum_{j=2^{i}-1}^{2^{i+1}-2} 2^{-i} \leq \sum_{j=2^{i}-1}^{2^{i+1}-2} 2^{-i}\left\|A_{n}\right\| \alpha_{j}\left(J_{n} A_{n}^{-1} I_{n} H_{n} Q_{n}\right)\left\|H_{n}^{-1}\right\| \\
\Rightarrow \quad 1 \leq\left\|A_{n}\right\| 2^{-i} \sum_{j=2^{i}-1}^{2^{i+1}-2} \alpha_{j}\left(J_{n} A_{n}^{-1} I_{n} H_{n} Q_{n}\right)\left\|H_{n}^{-1}\right\|
\end{gathered}
$$




$$
\Rightarrow \quad 1 \leq\left(\left\|A_{n}\right\|\left\|H_{n}^{-1}\right\|\right)^{p_{i}}\left(2^{-i} \sum_{j=2^{i}-1}^{2^{i+1}-2} \alpha_{j}\left(J_{n} A_{n}^{-1} I_{n} H_{n} Q_{n}\right)\right)^{p_{i}} .
$$

Therefore,

$$
\begin{aligned}
(n+1)^{\frac{1}{\sup _{i} p_{i}}} \leq L\left\|A_{n}\right\|\left\|H_{n}^{-1}\right\|\left[\sum_{i=0}^{n}\left(2^{-i} \sum_{j=2^{i}-1}^{i+1} \alpha_{j}\left(J_{n} A_{n}^{-1} I_{n} H_{n} Q_{n}\right)\right)^{p_{i}}\right]^{\frac{1}{\sup _{i} p_{i}}} \\
\Rightarrow \quad \frac{1}{\epsilon}(n+1)^{\frac{1}{\sup _{i} p_{i}}} \leq L\left\|A_{n}\right\|\left\|H_{n}^{-1}\right\| \frac{1}{\epsilon}\left[\sum_{i=0}^{n}\left(2^{-i} \sum_{j=2^{i}-1}^{2^{i+1}-2} \alpha_{j}\left(J_{n} A_{n}^{-1} I_{n} H_{n} Q_{n}\right)\right)^{p_{i}}\right]^{\frac{1}{\sup _{i} p_{i}}} \\
\Rightarrow \quad \frac{1}{\epsilon}(n+1)^{\frac{1}{\sup _{i} p_{i}}} \leq L\left\|A_{n}\right\|\left\|H_{n}^{-1}\right\| g\left(J_{n} A_{n}^{-1} I_{n} H_{n} Q_{n}\right) \\
\Rightarrow \quad \frac{1}{\epsilon}(n+1)^{\frac{1}{\sup _{i} p_{i}}} \leq L C\left\|A_{n}\right\|\left\|H_{n}^{-1}\right\|\left\|J_{n} A_{n}^{-1} I_{n} H_{n} Q_{n}\right\| \\
\Rightarrow \quad \frac{1}{\epsilon}(n+1)^{\frac{1}{\sup _{i} p_{i}}} \leq L C\left\|A_{n}\right\|\left\|H_{n}^{-1}\right\|\left\|J_{n} A_{n}^{-1}\right\|\left\|I_{n}\right\|\left\|H_{n} Q_{n}\right\| \\
\quad=L C\left\|A_{n}\right\|\left\|H_{n}^{-1}\right\|\left\|A_{n}^{-1}\right\|\left\|I_{n}\right\|\left\|H_{n}\right\| \\
\Rightarrow \quad \frac{1}{\epsilon}(n+1)^{\frac{1}{\sup _{i} p_{i}}} \leq 4 L C
\end{aligned}
$$

for some $L \geq 1$. Since $n$ is an arbitrary, this gives a contradiction. Thus $X$ and $Y$ both cannot be infinite dimensional when $S_{C(p)}^{\text {app }}(X, Y)=L(X, Y)$. This finishes the proof.

We express the accompanying theorem without verifications, these can be set up utilizing a standard procedure.

Theorem 6.4 If $\left(p_{n}\right)$ is an increasing bounded sequence with $p_{0}>1$, then the pre-quasiBanach operator ideal $S_{C(p)}^{\mathrm{Kol}}$ is small.

Corollary 6.5 If $1<p<\infty$, then the quasi-Banach operator ideal $S_{\mathrm{ces}_{p}}^{\mathrm{app}}$ is small.

Corollary 6.6 If $1<p<\infty$, then the quasi-Banach operator ideal $S_{\operatorname{ces}_{p}}^{\mathrm{Kol}}$ is small.

The following question arises naturally: for which $C(p)$, the pre-quasi-Banach ideal is simple?

Theorem 6.7 For any infinite dimensional Banach spaces $X, Y$, and for any bounded sequences $\left(p_{n}\right),\left(q_{n}\right)$ with $1<p_{n}<q_{n}$ for all $n \in \mathbb{N}$,

$$
L\left(S_{C(q)}, S_{C(p)}\right)=\Psi\left(S_{C(q)}, S_{C(p)}\right) .
$$

Proof Suppose that there exists $T \in L\left(S_{C(q)}, S_{C(p)}\right)$ which is not approximable. According to Lemma 2.1, we can find $A \in L\left(S_{C(q)}, S_{C(q)}\right)$ and $B \in L\left(S_{C(p)}, S_{C(p)}\right)$ with $B T A I_{k}=I_{k}$. Then 
it follows for all $k \in \mathbb{N}$ that

$$
\left\|I_{k}\right\|_{S_{C(p)}}=\sum_{i=0}^{\infty}\left(\frac{\sum_{j=2^{i}-1}^{2^{i+1}-2} s_{j}\left(I_{k}\right)}{2^{i}}\right)^{p_{i}} \leq\|B T A\|\left\|I_{k}\right\|_{S_{C(q)}} \leq \sum_{i=0}^{\infty}\left(\frac{\sum_{j=2^{i}-1}^{2^{i+1}-2} s_{j}\left(I_{k}\right)}{2^{i}}\right)^{q_{i}} .
$$

This contradicts Theorem (6.1). Hence $T \in \Psi\left(S_{C(q)}, S_{C(p)}\right)$, which finishes the proof.

Corollary 6.8 For any infinite dimensional Banach spaces $X$ and $Y$. If $1<p<q$, then

$$
L\left(S_{\operatorname{ces}_{q}}, S_{\operatorname{ces}_{p}}\right)=L_{C}\left(S_{\operatorname{ces}_{q}}, S_{\operatorname{ces}_{p}}\right)
$$

Theorem 6.9 If $\left(p_{n}\right)$ is an increasing bounded sequence with $p_{0}>1$, then the pre-quasiBanach space $S_{C(p)}$ is simple.

Proof Suppose that the closed ideal $L_{C}\left(S_{C(p)}\right)$ contains an operator $T$ which is not approximable. According to Lemma 2.1, we can find $A, B \in L\left(S_{C(p)}\right)$ with $B T A I_{k}=I_{k}$. This means that $I_{S_{C(p)}} \in L_{C}\left(S_{C(p)}\right)$. Consequently $L\left(S_{C(p)}\right)=L_{C}\left(S_{C(p)}\right)$. Therefore $\Psi\left(S_{C(p)}\right)$ is the only non-trivial closed ideal in $L\left(S_{C(p)}\right)$.

We give here the sufficient conditions such that the pre-quasi-ideal $S_{(C(p))_{\rho}}$ is approximable.

Theorem 6.10 Take any infinite dimensional Banach spaces $X$ and $Y$. If $\left(p_{n}\right)$ is an increasing bounded sequence with $p_{0}>1$, then $\overline{F(X, Y)}=S_{(C(p))_{\rho}}(X, Y)$, where $\rho(x)=$ $\sum_{i=0}^{\infty}\left(\frac{\sum_{j=2^{i}-1}^{2^{i+1}-2}\left|x_{j}\right|}{2^{i}}\right)^{p_{i}}$ for all $x \in C(p)$ and the converse is not necessarily true.

Proof Since $(C(p))_{\rho}$ is a pre-modular (sss), then from Theorem 2.10, we have $\overline{F(X, Y)}=$ $S_{(C(p))_{\rho}}(X, Y)$. Conversely since $I_{4} \in S_{(C(1))_{\rho}}$ the condition is not satisfied, which is a counter example. This finishes the proof.

\section{Eigenvalues of $s$-type operators}

We give here the sufficient conditions on $C(p)$ such that the pre-quasi-operator ideal formed by the sequence of $s$-numbers and this sequence space is equal the class of all bounded linear operators whose sequence of eigenvalues belongs to this sequence space.

\section{Notations 7.1}

$$
S_{E}^{\lambda}:=\left\{S_{E}^{\lambda}(X, Y) ; X \text { and } Y \text { are Banach Spaces }\right\},
$$

where

$$
\begin{aligned}
S_{E}^{\lambda}(X, Y):= & \left\{T \in L(X, Y):\left(\lambda_{n}(T)\right)_{n=0}^{\infty} \in E\right. \text { and } \\
& \left.\left\|T-\lambda_{n}(T) I\right\| \text { is not invertible for all } n \in \mathbb{N}\right\} .
\end{aligned}
$$

Theorem 7.2 Take any infinite dimensional Banach spaces $X$ and $Y$.If $\left(p_{n}\right)$ is an increasing bounded sequence with $p_{0}>1$, then

$$
S_{C(p)}^{\lambda}(X, Y)=S_{C(p)}(X, Y)
$$


Proof Let $T \in S_{C(p)}(X, Y)$, then $\left(s_{n}(T)\right)_{n=0}^{\infty} \in C(p)$. Since $\left(p_{n}\right)$ is an increasing bounded sequence with $p_{n}>1$ for all $n \in \mathbb{N}$, we have

$$
\sum_{i=0}^{\infty}\left(\frac{\sum_{j=2^{i}-1}^{2^{i+1}-2} x_{j}(T)}{2^{i}}\right)^{p_{i}} \geq \sum_{i=0}^{\infty}\left[s_{2^{i+1}-2}(T)\right]^{p_{i}} .
$$

Hence $\left(s_{n}(T)\right)_{n=0}^{\infty} \in \ell_{\left(p_{n}\right)}$, so $\lim _{n \rightarrow \infty} s_{n}(T)=0$. Suppose $\left\|T-s_{n}(T) I\right\|$ is invertible for all $n \in \mathbb{N}$, then $\left\|T-s_{n}(T) I\right\|^{-1}$ exists and is bounded for all $n \in \mathbb{N}$. This shows $\lim _{n \rightarrow \infty} \| T-$ $s_{n}(T) I\left\|^{-1}=\right\| T \|^{-1}$ exists and is bounded. Since $\left(S_{C(p)}, g\right)$ is a pre-quasi-operator ideal, we have

$$
I=T T^{-1} \in S_{C(p)}(X, Y) \quad \Rightarrow \quad\left(s_{n}(I)\right)_{n=0}^{\infty} \in C(p) \quad \Rightarrow \quad \lim _{n \rightarrow \infty} s_{n}(I)=0 .
$$

But $\lim _{n \rightarrow \infty} s_{n}(I)=1$. This is a contradiction, then $\left\|T-s_{n}(T) I\right\|$ is not invertible for all $n \in \mathbb{N}$. Therefore the sequence $\left(s_{n}(T)\right)_{n=0}^{\infty}$ is the eigenvalues of $T$.

Conversely, let $T \in S_{C(p)}^{\lambda}(X, Y)$, then $\left(\lambda_{n}(T)\right)_{n=0}^{\infty} \in C(p)$ and $\left\|T-\lambda_{n}(T) I\right\|=0$ for all $n \in \mathbb{N}$. This gives $T=\lambda_{n}(T) I$ for all $n \in \mathbb{N}$, then $s_{n}(T)=s_{n}\left(\lambda_{n}(T) I\right)=\left|\lambda_{n}(T)\right|$ for all $n \in \mathbb{N}$. Hence $\left(s_{n}(T)\right)_{n=0}^{\infty} \in C(p)$, so $T \in S_{C(p)}(X, Y)$. This finishes the proof.

\section{Acknowledgements}

This work was funded by the University of Jeddah, Saudi Arabia, under grant No. (UJ-02-048-DR). The authors, therefore, acknowledge with thanks the University technical and financial support. Also, the authors thank the anonymous referees for their constructive suggestions and helpful comments which led to significant improvement of the original manuscript of this paper.

\section{Funding}

Applicable.

\section{Availability of data and materials}

Not applicable.

\section{Competing interests}

The authors declare that they have no competing interests.

\section{Authors' contributions}

All authors contributed equally to the writing of this paper. All authors read and approved the final manuscript.

\section{Author details}

'University of Jeddah, College of Science and Arts at Khulis, Department of Mathematics, Jeddah, Saudi Arabia. ${ }^{2}$ Department of Mathematics, Faculty of Science, Ain Shams University, P.O. Box 1156, Cairo, 11566, Abbassia, Egypt.

${ }^{3}$ Department of Mathematics, Faculty of Education, Alzaeim Alazhari University, P.O. Box 1432, 13311 Khartoum, Sudan.

\section{Publisher's Note}

Springer Nature remains neutral with regard to jurisdictional claims in published maps and institutional affiliations.

Received: 12 February 2020 Accepted: 4 September 2020 Published online: 11 September 2020

\section{References}

1. Lim, K.P.: Matrix transformation on certain sequence space. Tamkang J. Math. 8(2), 213-220 (1977)

2. Lim, K.P.: Matrix transformation in the Cesáro sequence spaces. Kyungpook Math. J. 14, $221-227$ (1979)

3. Singh, R.K., Kumar, A.: Multiplication operators with closed range. Bull. Aust. Math. Soc. 16, 247-252 (1977)

4. Abrahmse, M.B.: Multiplication Operators. Lecture Notes in Mathematics, vol. 693, pp. 17-36. Springer, Berlin (1978)

5. Raj, K., Sharma, S.K., Kumar, A.: Multiplication operator on Musielak-Orlicz spaces of Bochner type. J. Adv. Stud. Topol. 3, 1-7 (2012)

6. Sharma, A., Raj, K., Sharma, S.K.: Products of multiplication composition and differentiation operators from $H^{\infty}$ to weighted Bloch spaces. Indian J. Math. 54, 159-179 (2012)

7. Singh, R.K., Manhas, J.S.: Composition Operators on Function Spaces. North-Holland, Amsterdam (1993)

8. Singh, R.K., Manhas, J.S.: Multiplication operators and composition operators with closed ranges. Bull. Aust. Math. Soc. $16,247-252(1977)$ 
9. Takagi, H., Yokouchi, K.: Multiplication and composition operators between two $L_{p}$-spaces. Contemp. Math. 232 321-338 (1999)

10. Bakery, A.A., Abou Elmatty, A.R.: Some properties of pre-quasi norm on Orlicz sequence space. J. Inequal. Appl. 2020, Article ID 55 (2020)

11. Illkhan, M., Demiriz, S., Kara, E.E.: Multiplication operators on Cesáro second order function spaces. Positivity 24, 605-614 (2020)

12. Kara, E.E., Zengin Alp, P.: A new class of operator ideals and approximation numbers. New Trends Math. Sci. 6(4), 8-15 (2018). https://doi.org/10.20852/ntmsci.2018.310

13. Zengin Alp, P., Kara, E.E.: Some equivalent quasinorms on $L_{\varphi}$, E. Facta Univ. (NIS), Ser. Math. Inform. 33(5), 739-746 (2018)

14. Bakery, A.A.: The spectrum generated by s-numbers and pre-quasi normed Orlicz-Cesáro mean sequence spaces. Open Math. 18, 846-857 (2020)

15. Mursaleen, M., Noman, A.K.: Compactness of matrix operators on some new difference sequence spaces. Linear Algebra Appl. 436, 41-52 (2012)

16. Komal, B.S., Gupta, S.: Multiplication operators between Orlicz spaces. Integral Equ. Oper. Theory 41(3), 324-330 (2001)

17. Komal, B.S., Pandoh, S., Raj, K.: Multiplication operators on Cesáro sequence spaces. Demonstr. Math. 49(4), 430-436 (2016)

18. Pietsch, A.: Operator Ideals. North-Holland, Amsterdam (1980)

19. Pietsch, A.: Small ideals of operators. Stud. Math. 51, 265-267 (1974)

20. Makarov, B.M., Faried, N.: Some properties of operator ideals constructed by s numbers. In: Theory of Operators in Functional Spaces, pp. 206-211. Academy of Science. Siberian Section, Novosibirsk (1977) (in Russian)

21. Faried, N., Bakery, A.A.: Small operator ideals formed by s numbers on generalized Cesáro and Orlicz sequence spaces. J. Inequal. Appl. 2018(1), Article ID 357 (2018). https://doi.org/10.1186/s13660-018-1945-y

22. Mrowka, T:. A brief introduction to linear analysis: Fredholm operators, geometry of manifolds, Fall 2004 (Massachusetts Institute of Technology: MIT OpenCouseWare) (2004)

23. Bakery, A.A., Mohammed, M.M.: Small pre-quasi Banach operator ideals of type Orlicz-Cesàro mean sequence spaces. J. Funct. Spaces 2019, Article ID 7265010 (2019)

24. Pietsch, A.: Eigenvalues and s-Numbers. Cambridge University Press, New York (1986)

25. Bakery, A.A., Mohammed, M.M.: Some properties of pre-quasi operator ideal of type generalized Cesáro sequence space defined by weighted means. Open Math. 17, 1703-1715 (2019). https://doi.org/10.1515/math-2019-0135

26. Altay, B., Başar, F.: Generalization of the sequence space $\ell(p)$ derived by weighted means. J. Math. Anal. Appl. 330(1), 147-185 (2007)

27. Pietsch, A.: Operator Ideals. VEB, Berlin (1978)

28. Pietsch, A.: s-Numbers of operators in Banach spaces. Stud. Math. 51, 201-223 (1974)

\section{Submit your manuscript to a SpringerOpen ${ }^{\circ}$ journal and benefit from:}

- Convenient online submission

- Rigorous peer review

- Open access: articles freely available online

- High visibility within the field

- Retaining the copyright to your article

Submit your next manuscript at $\boldsymbol{\nabla}$ springeropen.com 